\title{
Genetic background, recent advances in molecular biology, and development of novel therapy in Alport syndrome
}

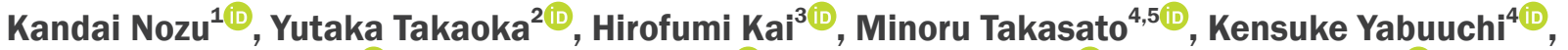

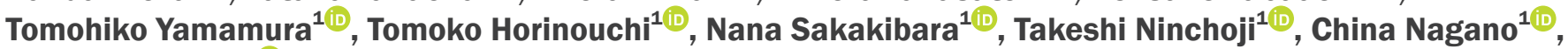 \\ Kazumoto lijima ${ }^{1 \text { iD }}$ \\ 'Department of Pediatrics, Kobe University Graduate School of Medicine, Kobe, Japan \\ ${ }^{2}$ Division of Medical Informatics and Bioinformatics, Kobe University Hospital, Kobe, Japan \\ ${ }^{3}$ Department of Molecular Medicine, Graduate School of Pharmaceutical Sciences, Kumamoto University, Kumamoto, Japan \\ ${ }^{4}$ Laboratory for Human Organogenesis, RIKEN Center for Biosystems Dynamics Research, Kobe, Japan \\ 5Laboratory of Molecular Cell Biology and Development, Department of Animal Development and Physiology, Graduate School of \\ Biostudies, Kyoto University, Kyoto, Japan
}

\begin{abstract}
Alport syndrome (AS) is a progressive inherited kidney disease characterized by hearing loss and ocular abnormalities. There are three forms of AS depending on inheritance mode: X-linked Alport syndrome (XLAS), autosomal recessive AS (ARAS), and autosomal dominant AS (ADAS). XLAS is caused by pathogenic variants in COL4A5, which encodes type IV collagen $\alpha 5$ chain, while ADAS and ARAS are caused by variants in COL4A3 or COL4A4, which encode type IV collagen $\alpha 3$ or $\alpha 4$ chain, respectively. In male XLAS or ARAS cases, end-stage kidney disease (ESKD) develops around a median age of 20 to 30 years old, while female XLAS or ADAS cases develop ESKD around a median age of 60 to 70 years old. The diagnosis of AS is dependent on either genetic or pathological findings. However, determining the pathogenicity of the variants detected by gene tests can be difficult. Recently, we applied the following molecular investigation tools to determine pathogenicity: 1) in silico and in vitro trimer formation assay of $\alpha 345$ chains to assess triple helix formation ability, 2) kidney organoids constructed from patients' induced pluripotent stem cells to identify $\alpha 5$ chain expression on the glomerular basement membrane, and 3) in vitro splicing assay to detect aberrant splicing to determine the pathogenicity of variants. In this review article, we discuss the genetic background and novel assays for determining the pathogenicity of variants. We also discuss the current treatment approaches and introduce exon skipping therapy as one potential treatment option.
\end{abstract}

Keywords: Alport syndrome, Glomerular basement membrane, Induced pluripotent stem, Splicing

Received June 26, 2020; Revised August 6, 2020; Accepted August 8, 2020

Editor: Tae-Hyun Yoo, Yonsei University, Seoul, Republic of Korea

Correspondence: Kandai Nozu

Department of Pediatrics, Kobe University Graduate School of Medicine, 7-5-1 Kusunoki-cho, Chuo, Kobe, Hyogo 650-0017, Japan. E-mail: nozu@med.kobe-u.ac.jp

Copyright (C) 2020 by The Korean Society of Nephrology

(a) This is an open-access article distributed under the terms of the Creative Commons Attribution Non-Commercial License (http://creativecommons.org/ licenses/by-nc-nd/4.0/), which permits unrestricted non-commercial use, distribution, and reproduction in any medium, provided the original work is properly cited. 


\section{Introduction}

Alport syndrome (AS) is a hereditary condition characterized by progressive kidney disease, sensorineural hearing loss, and ocular abnormalities [1]. AS is caused by a defect in one of three type IV collagen alpha chains due to a pathogenic variant of the genes encoding these proteins: type IV collagen $\alpha 3$ chain [ $\alpha 3($ IV)], encoded by COL4A3; $\alpha 4$ chain [ $\alpha 4$ (IV)], encoded by COL4A4; and $\alpha 5$ chain $[\alpha 5(\mathrm{IV})]$, encoded by COL4A5. There are three types of AS, depending on the mode of inheritance: Xlinked AS (XLAS), autosomal recessive AS (ARAS), and autosomal dominant AS (ADAS). XLAS is caused by $\mathrm{CO}$ L4A5 gene variants, whereas ARAS and ADAS are caused by $C O L 4 A 3$ or COL4A4 gene variants. Male XLAS and ARAS patients develop end-stage kidney disease (ESKD) at a mean age of around 20 to 30 years [2-6], whereas in female XLAS and ADAS cases this occurs at around 60 to 70 years [7-9]. Male XLAS cases show a very strong genotype-phenotype correlation, whereas this correlation
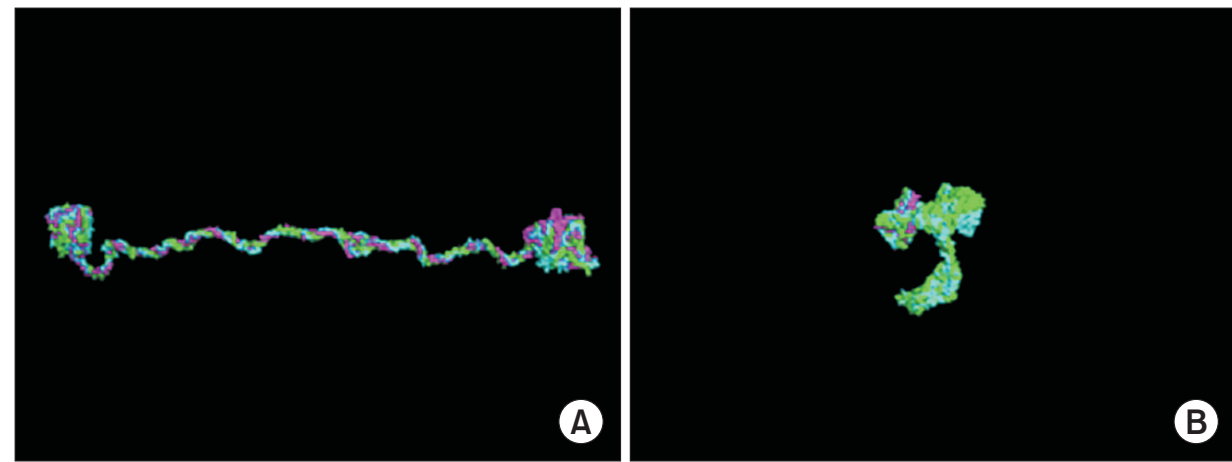

Figure 1. Three-dimensional (3D) structure analysis of collagen triple helix. Type IV collagen $\alpha 345$ chains form a triple helix. This $3 D$ structure was constructed by supercomputer K using homology modeling. (A) Wild-type structure. (B) Nonsense mutation in COL4A5.
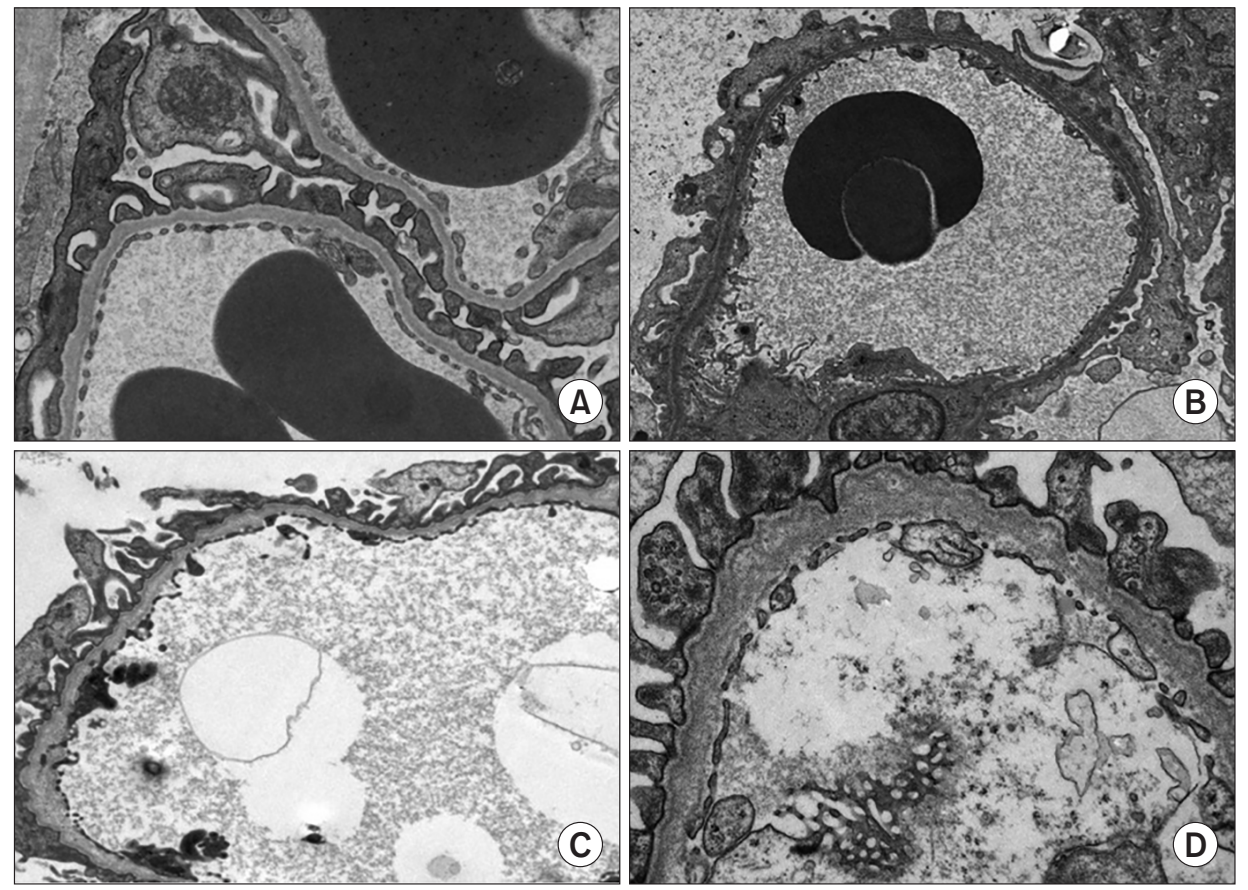

Figure 2. Glomerular basement membrane (GBM) change in Alport syndrome (AS) observed by electron microscopy. (A) Normal GBM showing three layers. (B) Thin basement membrane, which is typically observed in milder cases, including female X-linked AS and autosomal dominant AS. (C) Diffuse thinning and splitting of the lamina densa, which are findings specific to AS but observed in relatively mild cases or early stages of AS. (D) Diffuse thickening and lamellation, which are findings specific to AS. 
is weak in ARAS cases and absent in female XLAS and ADAS cases. In addition, some XLAS cases show atypical mild phenotypes, but in most cases, the factors leading to the milder phenotypes can be clarified by identifying the genetic background, such as missense variants, in-frame deletion variants, or somatic mosaic variants [1]. One of these atypical cases yielded findings that led to the development of exon skipping therapy, a gene-targeted therapy, for male XLAS cases with truncating variants that showed severe phenotypes [10]. In this review article, we discuss the genetic background of AS, novel assays for determining the pathogenicity of variants, and current treatment options; we also introduce exon skipping therapy that we are developing and that has been confirmed to be very effective against severe XLAS cases, at least in animal models [10]. In addition, we are attempting to construct kidney organoids from induced pluripotent stem (iPS) cells, not only to identify the $\alpha 5$ (IV) expression pattern in the glomerular basement membrane (GBM), but also in an attempt to establish a model to assess the novel treatment effects.

\section{Molecular onset mechanisms for AS}

$\alpha 3(\mathrm{IV}), \alpha 4$ (IV), and $\alpha 5$ (IV) form a triple helix (Fig. 1A) that combines tightly with other triple helices to form the GBM. If one of the three $\alpha$ chains becomes defective from a pathogenic variant of the encoding gene, the normally highly ordered GBM gradually breaks down, including splitting of the lamina densa in GBM, which is referred to as the basket weave change (Fig. 2). These changes accelerate the glomerular sclerotic changes and lead to kidney dysfunction. Immunostaining of the glomerulus revealed that $\alpha 5$ (IV) is normally detected in both GBM and Bowman's capsule. However, male XLAS cases show complete negativity for $\alpha 5$ (IV) expression, while female XLAS cases show $\alpha 5$ (IV) expression with a mosaic pattern; ARAS cases show $\alpha 5$ (IV) expression only in Bowman's capsule; and ADAS cases show normal $\alpha 5$ (IV) expression (Fig. 3). We described the molecular mechanisms behind these expression patterns in a previous review article [1]. The $\alpha 5$ (IV) expression patterns can be explained by the lack of production of $\alpha 3$ (IV), $\alpha 4$ (IV),
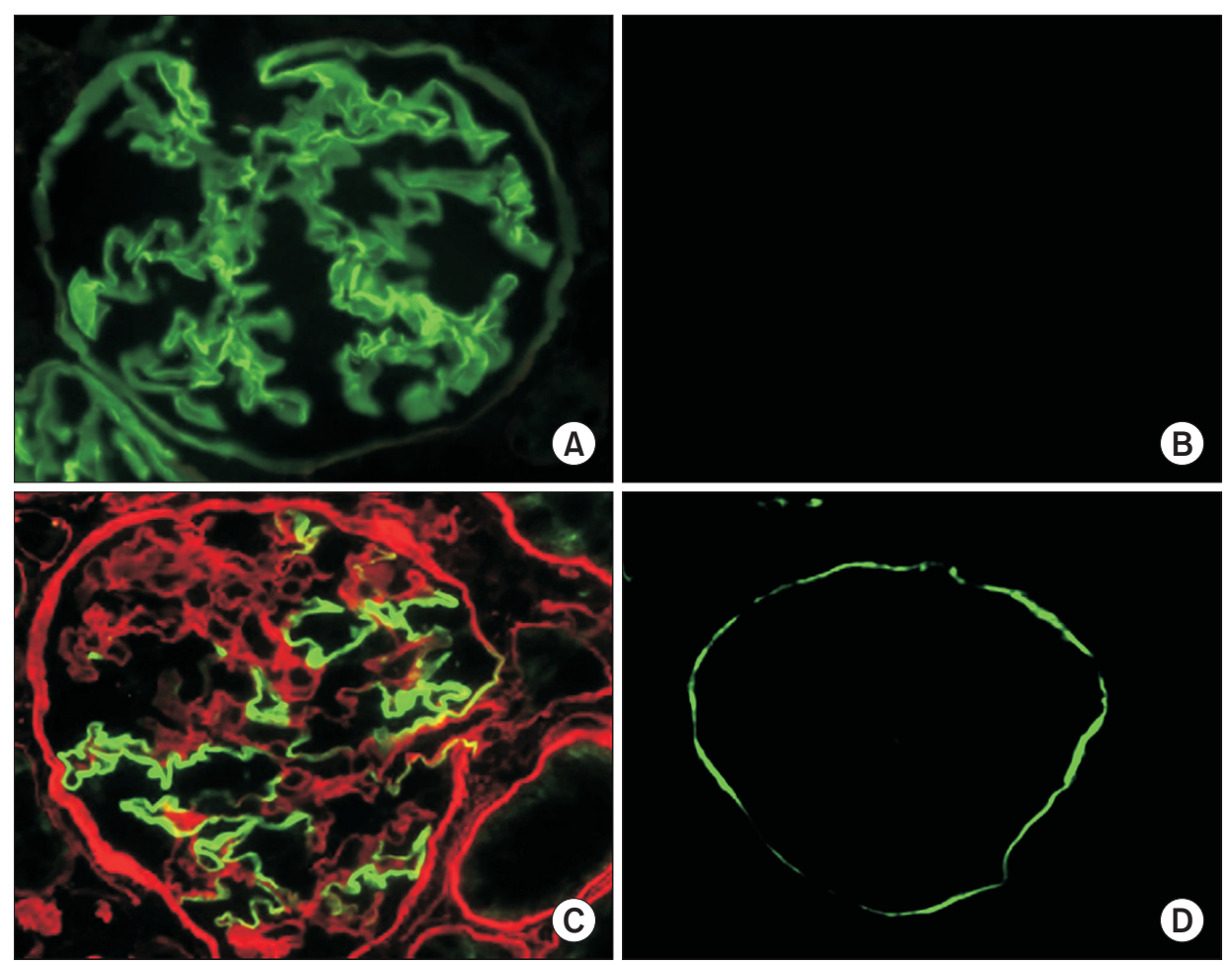

Figure 3. Immunohistochemical analysis of type IV collagen $\alpha 5$ chain in glomerulus. (A) Normal control shows full expression in both glomerular basement membrane (GBM) and Bowman's capsule (BC). (B) A male X-linked Alport syndrome (XLAS) case shows completely negative expression in both GBM and BC. (C) A female XLAS case shows a mosaic pattern of expression in both GBM and BC due to the mechanisms of X-chromosome inactivation that occur in female cells. (D) An autosomal recessive AS case shows negative expression only on GBM, but positivity on BC, because BC consists of the $\alpha 556$ triple helix. 
or $\alpha 5$ (IV), resulting in the failure of $\alpha 345$ (IV) trimer formation (Fig. 1B). However, there are male XLAS cases with atypical expression of $\alpha 5$ (IV). We found that $\alpha 5$ (IV) expression was confirmed in $29 \%$ of such cases. These $\alpha 5$-positive cases show clearly milder phenotypes than typical cases with negative $\alpha 5$ (IV) expression. All of these cases with $\alpha 5$-positive expression in GBM possessed nontruncating (missense or in-frame deletion) mutations or somatic mosaic mutations $[11,12]$. Therefore, for some non-truncating pathogenic variants, $\alpha 345$ (IV) trimer can be produced, although its structure does not completely match the normal form. We discuss this point later in this review.

\section{Overall genetic background in AS}

We recently published the results of genetic testing for AS in Japan [13]. We conducted Sanger sequencing for 294 suspected AS cases and next-generation sequencing (NGS) for 147 suspected AS cases. The results showed that 239 cases in the Sanger group (81\%) and 126 (86\%) in the NGS group were genetically diagnosed with AS by direct sequencing methods. In addition, in 23 cases, copy number variations (CNV) were detected by pair analysis and/or multiplex ligation-dependent probe amplification (MLPA), and in eight cases, pathogenic single-base substitutions in introns were detected by RNA sequencing performed to detect aberrant splicing caused by these variants. In contrast, two cases were diagnosed with other inherited diseases: one with Brachio-oto-renal syndrome in whom nephropathy and hearing loss were observed and EYA1 gene heterozygous mutation was detected, and the other with NPHS1-related nephropathy who was showing hematuria, heavy proteinuria and pathologically thin basement membrane by electron microscopy and compound heterozygous gene variant in NPHS1. NPHS1 gene variants usually lead to a more severe phenotype of congenital nephrotic syndrome; however, some missense variants lead to milder phenotypes, such as in our case [14]. The overall diagnosis rate was $90 \%$ in this study [13].

The proportions of the three modes of inheritance of XLAS, ARAS, and ADAS are $80 \%, 15 \%$, and $5 \%$, respectively [1]. However, our recent study reported proportions of $74 \%$, $9 \%$, and $17 \%$, respectively (Fig. $4 \mathrm{~A}$ included patients with no variant detection; XLAS: $67 \%$, ADAS: $15 \%$, ARAS: 8\%, and no detection: $10 \%$ ) [13]. This revealed that there are considerably more ADAS cases than previously considered. Of note, from our experience, adult cases with chronic kidney disease (CKD) accompanied by hematuria should be suspected as having ADAS when they were pathologically denied of having IgA nephropathy [15].

Among COL4A5 gene variants, $52 \%$ were missense variants, $8 \%$ were nonsense variants, $13 \%$ were splicing consensus variants, and $17 \%$ were small arrangements including insertions, deletions, and duplications. These variants can be detected by sequencing analysis. In contrast, $7 \%$ involved CNVs, usually detected by MLPA or pair analysis, and 3\% involved deep intronic variants leading to aberrant splicing, as detected by RNA sequencing (Fig. 4B) [16-19]. Pair analysis is the NGS data-dependent CNV detection method that compares per base interval read depth of the wild-type form and patient. The reduc-

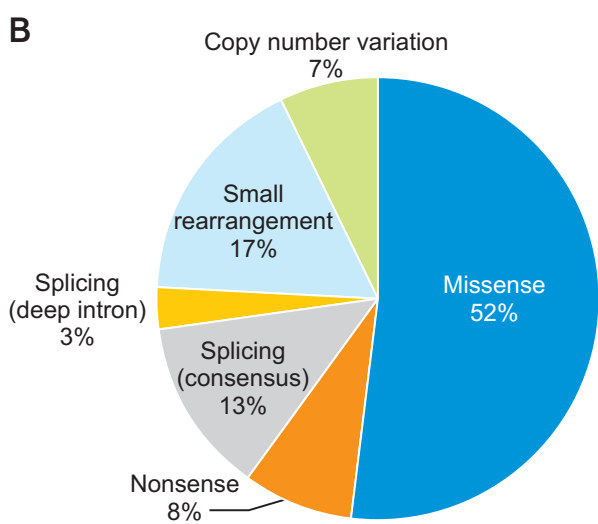

Figure 4. Genetic background in Alport syndrome (AS). (A) Overall genetic test results for suspected Alport syndrome cases $(n=441)$. (B) Variant types observed in X-linked AS (XLAS) cases ( $n=295$ ). ADAS, autosomal dominant AS; ARAS, autosomal recessive AS. 
tion of depth by about half in two consequent exons is suspected to be a heterozygous deletion that should be confirmed by MLPA [17].

\section{Triple helix formation assay}

In recent studies, we developed in silico and in vitro assays to examine the influence of variants in COL4A genes on the ability of the proteins to form a triple helix $[10,20,21]$.

\section{In silico three-dimensional (3D) structure analysis of} collagen triple helix

We modeled the 3D structure of the $\alpha 345$ (IV) trimer using homology modeling [10,20,21]. Fig. 1A shows the triple helix of $\alpha 345(\mathrm{IV})$ by the modeling approach. With this modeling approach, we can input information about variants and see the resulting changes of the trimer structure. Fig. 1B shows the trimer formation associated with COL4A5 nonsense mutation in exon 21, with its structure having completely collapsed. Using this approach, we showed that a noncollagenous (NC) domain encoded at the 3' end of the COL4A5 gene is essential for trimer formation [10]. We are now studying whether this approach can reflect the clinical severity of pathogenic variants. For example, missense variants that change Gly to another amino acid in COL4A5 are always considered to be pathogenic. However, p.Gly953Val is considered to be nonpathogenic [22] and p.Glyl000Val leads to a very mild phenotype resembling benign familial hematuria [23]. We are now attempting to reveal these differences using this approach.

In vitro split luciferase-based trimer formation of $\alpha 345(I V)$ protein assay

We have also established a split nanoluciferase (NanoLuc) complementation system to examine the formation of the $\alpha 345$ (IV) trimer in vitro [20,21]. This system is composed of a large fragment and a small fragment of NanoLuc luciferase. Luminescence is observed when the split NanoLuc-tagged proteins interact with large and small fragments. We constructed plasmids containing full length of cDNA for COL4A3, COL4A4, and COL4A5, separately, and C-terminal was tagged by a small fragment for
COL4A3 and by a large fragment for COL4A5 (Fig. 5A). These plasmids are transfected into HEK293T cells and luminescence is detected with high sensitivity from the trimer of $\alpha 345$ (IV), but not from the homodimer- or heterodimer-expressing cells or supernatant (Fig. 5B). Using this assay, we revealed a strong correlation of the cellbased a345(IV) trimer formation with clinical severity [20] and anticipate the ability to distinguish pathogenic variants from nonpathogenic ones or predict the kidney prognosis. We also used this assay to show that $\alpha 5$ (IV) with missense variants can form the $\alpha 345$ (IV) trimer and be excreted from cells. These results are compatible with the finding that $\alpha 5(\mathrm{IV})$ is expressed in some male XLAS cases with missense variants that tend to show milder phenotypes $[11,12]$.

\section{Generation of kidney organoids from patients' iPS cells}

As is caused by genetic mutations of collagen genes, it is possible to reproduce the phenotypes of AS in kidney generated from AS patient-derived iPS cells [10]. Currently, there are protocols in which human iPS cells are differentiated into self-organizing kidney-like tissue in vitro, so-called kidney organoids [24-26]. In our protocol, kidney organoids include nephrons, collecting ducts, renal stromal cells, and endothelium [27] and develop GBM and slit-diaphragm structures in glomeruli when

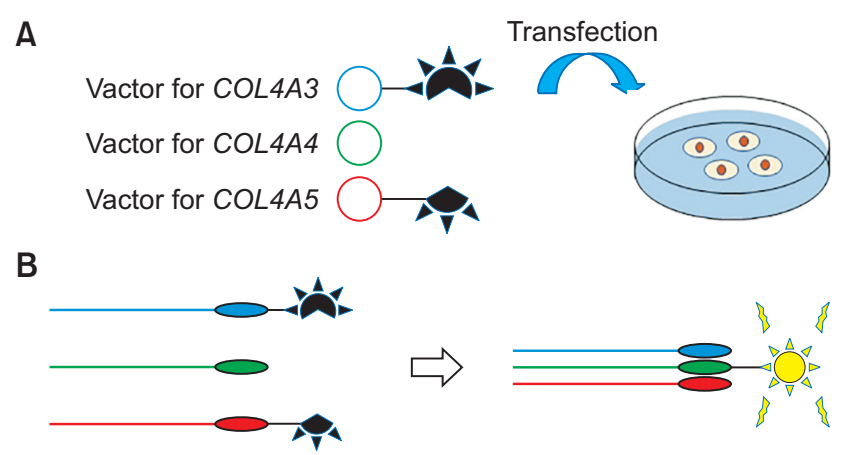

Figure 5. Split luciferase-based trimer formation of the $\alpha 345$ (IV) protein assay. (A) A split nanoluciferase (NanoLuc) complementation system to examine the formation of the $\alpha 345$ (IV) trimer in vitro. Vectors containing type IV collagen $\alpha 345$ sequences are transfected separately into cultured cells. (B) This assay is composed of a large fragment and a small fragment of NanoLuc luciferase. Luminescence is observed when a trimer of three proteins forms and the split NanoLuc-tagged proteins interact with the large and small fragments. 
they are cultured in vivo by transplantation into animals [28]. We also confirmed the expression of $\alpha 5$ (IV) in GBM of kidney organoids (Fig. 6). CRISPR/Cas9 genome-editing technology enables scientists to rapidly and reliably create desired genetic mutations of collagen genes in human iPS cells or to correct causative gene mutations in AS patient-derived iPS cells to obtain isogenic control cell lines. However, to the best of our knowledge, there have been no reported studies on AS in which degraded GBM was successfully reproduced in kidney organoids using patients' iPS cells. However, such de novo technologies will enable us to investigate the development of AS in vitro.

\section{In vitro splicing assay for detecting splicing abnormalities}

RNA sequencing is often difficult because of the low expression levels of transcripts in peripheral leukocytes, fragility of transcripts, or reduced transcript expression because of nonsense-mediated decay for truncating variants. Recently, we and other groups developed an in vitro splicing assay using a minigene construct to detect aberrant splicing caused by variants in COL4A5 (Fig. 7) $[16,29-31]$. This assay can easily detect the pathogenicity of single-base substitutions causing aberrant splic-
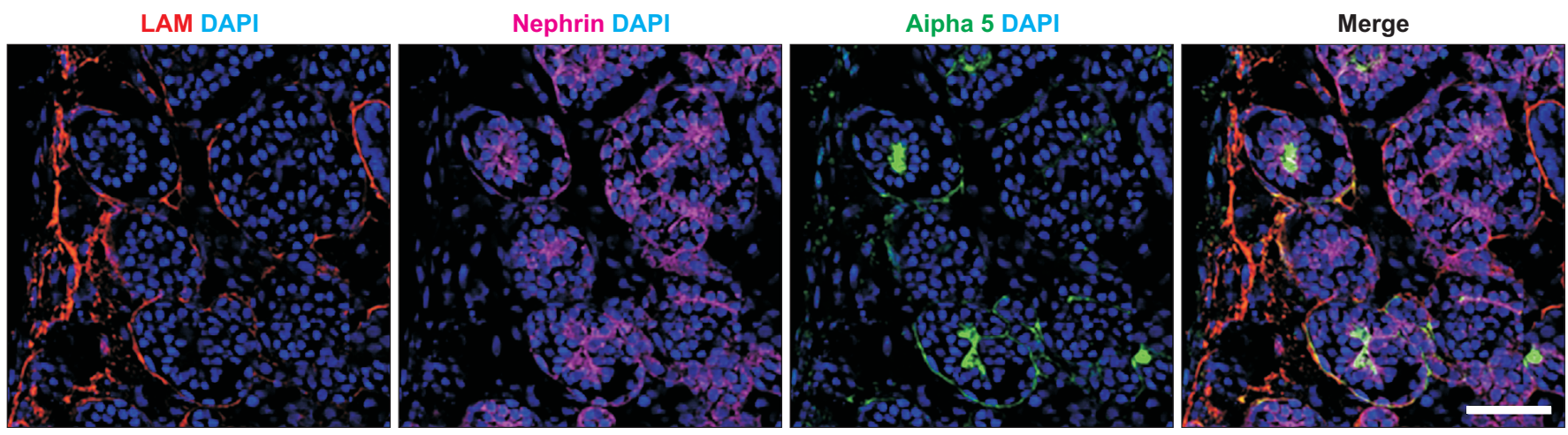

Figure 6. Glomeruli in kidney organoids express COL4A5. Glomeruli containing podocytes (Nephrin ${ }^{+}$) and deposited basement membrane $\left(\mathrm{LAM}^{+}\right)$clearly express Type IV collagen alpha 5. Cell nuclei were stained with 4',6-diamidino-2-phenylindole (DAPI). Scale bar, $50 \mu \mathrm{m}$.

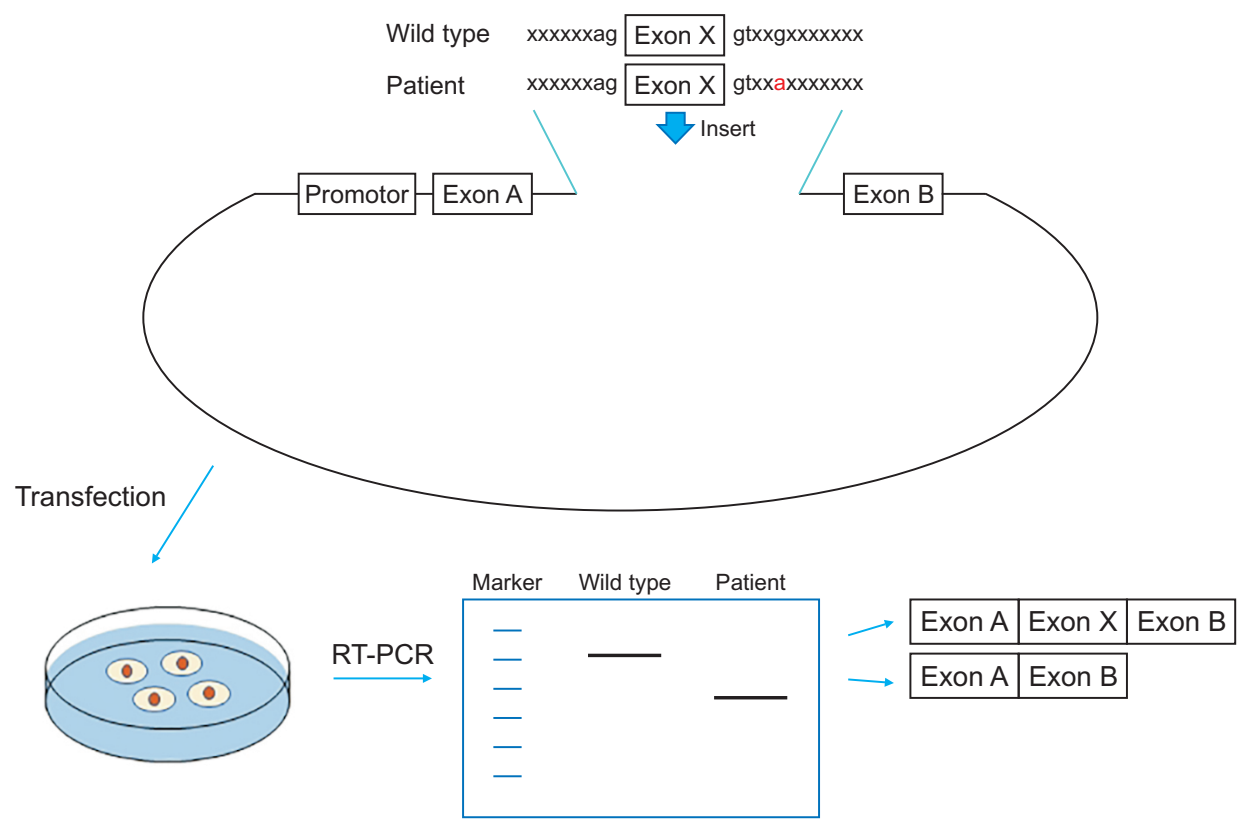

Figure 7. In vitro splicing assay for detecting splicing abnormalities. A wild-type or patient sequence containing a suspected splicing variant is inserted into a minigene and transfected into cultured cells. After 24 hours, transcripts are extracted and reverse transcript polymerase change reaction (RT-PCR) is conducted to determine whether the variants actually cause aberrant splicing. 
ing in exons and introns, such as synonymous variants or variants outside of the intronic consensus sequences $[16,30,32,33]$. Here we describe one example of such detection (Fig. 7) in a XLAS case with a single-base substitution of IVS $\mathrm{X}+5 \mathrm{G}>\mathrm{A}$ (the fifth nucleotide in intron $\mathrm{X}$ is mutated from $\mathrm{G}$ to $\mathrm{A}$ ). Without transcript analysis, we cannot determine whether this variant is pathogenic. Thus, we can construct a vector containing a promoter, Exon A, and Exon B; in addition, a sequence containing exon $\mathrm{X}$ in conjunction with nearby intronic sequences harboring wild-type or the variant is inserted between Exons A and B. This vector is called a minigene construct. This vector is transfected into cultured cells and subsequently produces transcripts. The transcripts are extracted and reverse transcript polymerase change reaction is conducted to check whether the variant leads to aberrant splicing. This assay is very effective for screening the pathogenicity of splicing variants [16].

\section{Genotype-phenotype correlations in AS}

\section{Male XLAS}

Three reports have described the correlations between genotype and phenotype in male XLAS patients [2-4]. All of these reports describe common results of cases with truncating variants (nonsense, small rearrangement, and large rearrangement) that developed ESKD more than 10 years earlier than cases with non-truncating variants (missense and in-frame small deletion). In addition, with small deletion variants in which the number of deleted nucleotides is a multiple of 3 , the cases tend to show milder phenotypes because each triplet of nucleotides encodes an amino acid. When the number of deleted nucleotides is a multiple of 3 , an in-frame deletion occurs and the rest of the amino acid sequence remains unchanged. In contrast, cases with splice site variants have been reported to show intermediate severity of developing ESKD at the median age between that for truncating and non-truncating variants [2-4]. We further investigated the correlation of phenotypes and the number of deleted nucleotides at the transcript level, concentrating on splicing variants. For all cases suspected of having aberrant splicing due to splicing variants, we conducted RNA sequencing and revealed the aberrant splicing. We divided the cases into two groups of truncating mutations (in which the deleted nucleotide number was not a multiple of $3 ; n=21$, from 14 families) and non-truncating mutations (in which the deleted nucleotide number was multiple of $3 ; \mathrm{n}=25$, from 15 families) at the transcript level. The results showed that the median age for developing ESRD was 20 years for patients with truncating mutations and 29 years for those with non-truncating ones $(P=0.001)[16]$. These results suggest that, even in cases with splicing abnormalities, there is a strong genotypephenotype correlation.

\section{Female XLAS}

Regarding female XLAS cases, no genotype-phenotype correlations were observed in two previous studies $[7,9]$. Some findings suggested that an uneven pattern of Xchromosome inactivation (i.e., skewed X-chromosome inactivation) would determine the severity of XLAS in females [34,35]. However, to date, no study has systematically demonstrated this correlation.

\section{ARAS}

There are two previous reports and one systemic review article discussing the genotype-phenotype correlation in ARAS $[5,6,36]$. Storey et al [36] reported that patients with truncating mutations in at least one allele showed a more severe phenotype with early onset of renal failure compared with patients without truncating mutations. However, our group reported that no genotype-phenotype correlation was observed in a Japanese ARAS cohort [6]. Finally, Lee et al [5] conducted a systematic review of 148 previously reported cases and concluded that there was a genotype-phenotype correlation according to the number of missense mutations. Patients with two missense mutations had delayed onset of ESKD and rarely showed sensorineural hearing loss.

\section{ADAS}

To the best of our knowledge, no genotype-phenotype correlations have yet been observed in ADAS. Even within one family sharing the same variant, the clinical severity differed significantly [8]. In a recent study with a large cohort of CKD patients, COL4A3 or COL4A4 gene variants contributed to $16 \%$ of 312 cases with CKD [37]. Although 
we reported that $17 \%$ of the AS cases are ADAS, the cohort study suggested that far more CKD cases caused by ADAS are undiagnosed. The diagnosis of ADAS is difficult as such cases always lack pathological features typical of AS [15]. Therefore, it is important for nephrologists to recognize this condition and perform genetic analysis for appropriate patients. The factors determining the severity of ADAS are still unclear and can include modifier genes, other coinciding renal diseases, or other acquired factors such as hypertension, diabetes, smoking, and obesity.

The disease spectrum of ADAS is still currently under discussion. Historically, cases with only hematuria and having heterozygous variants in either $C O L 4 A 3$ or $C O$ $L 4 A 4$ were classified with thin basement membrane disease (TBMD). In fact, most of these cases will not develop ESKD and the clinical course is benign, which is different from conventional AS. However, with the development of recent molecular diagnostic techniques, many CKD cases were revealed to be heterozygous COL4A3 or COL4A4 gene variant carriers $[15,37]$ and they were not always benign. In addition, some of the cases diagnosed with TBMD in their early life will develop into ESKD in their later life. Therefore, recent review articles have suggested that all cases with variant carriers in these genes should be classified into autosomal AS $[1,38,39]$. This classification can avoid ADAS patients missing initiation of treatment.

\section{Treatment}

There is currently no radical therapy for AS; however, treatment by renin-angiotensin system inhibitors (RAS inhibitors) has been performed to reduce proteinuria and delay progression to renal failure using nephroprotective drugs. The results of two randomized controlled trials (RCTs) revealed the reduction of urine protein levels by angiotensin-converting enzyme inhibitors (ACEIs) and angiotensin receptor blockers in AS [40,41]. A large retrospective study reported that ACEIs can delay the progression to ESRD in AS [42]. The results of a RCT (EARLY PRO-TECT ALPORT study) confirming the nephroprotective effects have recently been published. These results showed that ramipril treatment reduced the risk of disease progression by almost half (hazard ratio, 0.51) [43]. The study strongly recommended treatment with an RAS inhibitor for AS.
Some new drugs for AS have entered clinical trials. Bardoxolone methyl is in a phase II/III trial (CARDINAL study). Bardoxolone methyl works as an activator for the KEAP1-Nrf2 pathway and blocks the NF- $\mathrm{B}$ pathway, activating many anti-inflammatory or antioxidant genes. A recent RCT for diabetic kidney disease patients as a phase 2 clinical trial (TSUBAKI study) showed a significant increase of measured glomerular filtration rate [44]. RG-012 (also known as lademirsen, which interferes with microRNA-21 (miR-21) interference) is under a phase II trial. miR-21 is involved in the progression of fibrogenic diseases. Inhibiting miR-21 by oligonucleotides improved the survival and histological findings in AS mice [45].

\section{Exon skipping therapy}

Our group is developing exon skipping therapy using antisense oligonucleotide (ASO) for treatment of severe male XLAS cases. This approach replaces the truncating variants with a non-truncating in-frame deletion mutation at the transcript level, which leads to milder phenotypes in AS. ASO binds to the exonic splicing enhancer region and, as a result, this exon is not recognized as an exon in the splicing process, leading to exon skipping (Fig. $8 \mathrm{~A})$. As described above, when the number of skipped nucleotides is a multiple of 3 , the severe phenotype from a nonsense mutation is changed to a milder phenotype from an in-frame deletion. Among the exons in the collagenous domain of the COL4A5 gene (exons 3 to 46), 35 exons out of 44 consist of nucleotides that are a multiple of 3. Therefore, theoretically, if patients have truncating variants in one of these exons, exon skipping therapy could replace truncating variants with non-truncating variants. In these cases, exon skipping therapy would lead to establishment of the NC domain in $\alpha 5$ (IV) (Fig. $8 \mathrm{~B})$, which allows the formation of a trimer with $\alpha 3$ (IV) and $\alpha 4$ (IV), leading to a milder phenotype (Fig. 8C). We conducted an in vivo study using an AS mouse model possessing a nonsense mutation in exon 21 of col4a 5 [46] and treated mice with ASO leading to exon 21 (84 bp) skipping. This treatment had a remarkable effect of improving the pathological findings with the recovery of $\alpha 5$ (IV) chain expression, reduced urine protein excretion level, delayed development of ESKD, and extension of survival [10]. To date, we have only developed ASO for exon 21, so further work is needed to develop this ther- 
A

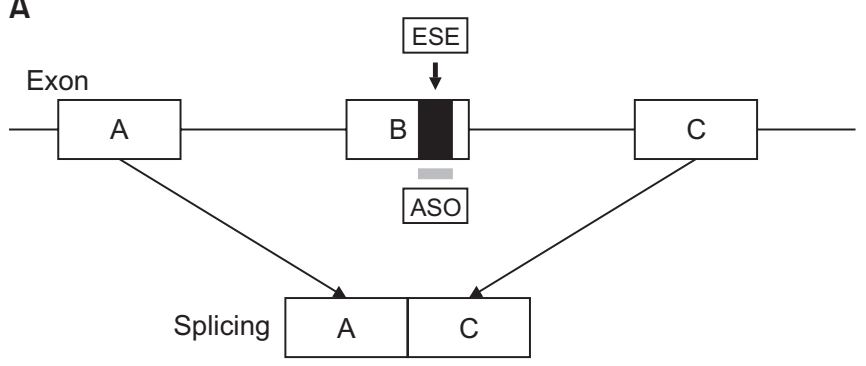

C

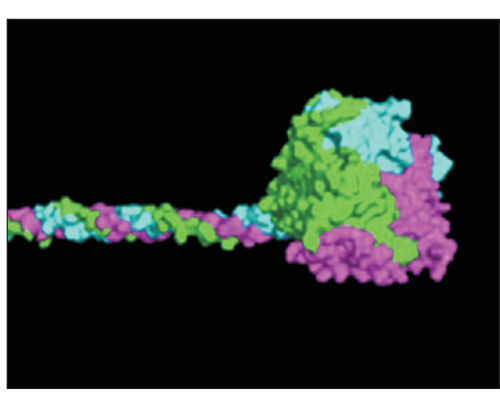

apy to target other exons. We are currently attempting to establish a model to assess the effect of this treatment for other exons using kidney organoids. We confirmed that organoids from a patient's iPS cells with a truncating variant in COL4A5 gene do not express $\alpha 5$ (IV). Our experiments to determine when and how often ASO should be injected to AS patients are ongoing. We will then conduct safety evaluation tests using animals and proceed to a clinical trial.

\section{Conclusion}

Recent developments in genetic studies and related in silico and in vitro analyses in AS have led to accurate diagnoses, investigation of the genetic background including genotype-phenotype correlation, and the development of novel therapies. Additional work in this field could help AS patients by further improving their quality of life.

\section{Conflicts of interest}

This study was supported by Grants-in-Aid for Scientific Research (KAKENHI) from the Ministry of Education, Culture, Sports, Science and Technology of Japan (subject ID: 19K08726 to Kandai Nozu, 16K19642 to Tomohiko Yamamura, and 26293203 and 17 H04189 to Kazumoto Iijima); the Japan Agency for Medical Research and Devel-
B

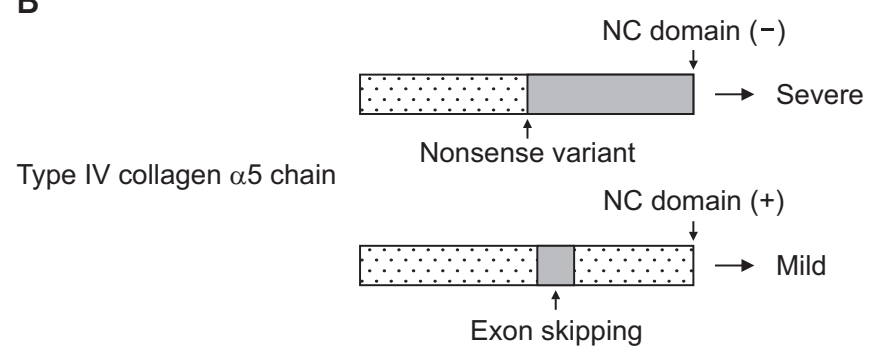

Figure 8. Exon skipping therapy. (A) Antisense oligonucleotide (ASO) designed to bind to exonic splicing enhancer (ESE) disturbs the recognition of an exon in the splicing process. (B) As a result, ASO leads to an exon-skipped transcript and produces an in-frame deleted protein possessing a noncollagenous (NC) domain. (C) The $\mathrm{NC}$ domain is the critical part for trimer formation by type IV collagen a345 chains.

opment (AMED) (Grant Number JP19ek0109231h0003 to Kandai Nozu and Kazumoto Iijima, 19ek0109231s0103 to Hirofumi Kai, and 19ek0109231s0203 to Minoru Takasato); and MEXT/JSPS Grants-in-Aid for Scientific Research (C) (Grant Number 18K07414 to Yutaka Takaoka).

Kandai Nozu and Kazumoto Iijima have filed a patent application on the development of antisense nucleotides for exon skipping therapy in Alport syndrome. Hirofumi Kai holds a patent related to the trimerization assay (Japanese Patent Application No. 2017-99497).

This research used computational resources of the $\mathrm{K}$ supercomputer provided by the RIKEN Center for Computational Science through the HPCI System Research project (Project ID: hp180288, Yutaka Takaoka and Kandai Nozu).

Kandai Nozu has received consulting fees from Kyowa Kirin Co., Ltd. and lecture fees from Kyowa Kirin Co., Ltd., Novartis Pharmaceuticals Corporation, and Chugai Pharmaceutical Co., Ltd. Kazumoto Iijima has received grant support from Daiichi Sankyo Co., Ltd., consulting fees from Kyowa Kirin Co., Ltd. and Boehringer Ingelheim, and lecture fees from Kyowa Kirin Co., Ltd., Chugai Pharmaceutical Co., Ltd., Takeda Pharmaceutical Company, Integrated Development Associates, and Novartis Pharmaceuticals Corporation. 


\section{Authors' contributions}

Kandai Nozu organized the study and wrote the manuscript. Yutaka Takaoka established the in silico 3D structure analysis of the collagen triple helix. Hirofumi Kai established the in vitro split luciferase-based trimer formation of the $\alpha 345$ (IV) protein assay. Minoru Takasato and Kensuke Yabuuchi are establishing kidney organoids from patient induced pluripotent stem cells. Tomohiko Yamamura, Tomoko Horinouchi, Nana Sakakibara, Takeshi Ninchoji, China Nagano, and Kazumoto Iijima are developing the exon skipping therapy.

\section{References}

[1] Nozu K, Nakanishi K, Abe Y, et al. A review of clinical characteristics and genetic backgrounds in Alport syndrome. Clin Exp Nephrol 2019;23:158-168.

[2] Bekheirnia MR, Reed B, Gregory MC, et al. Genotype-phenotype correlation in X-linked Alport syndrome. J Am Soc Nephrol 2010;21:876-883.

[3] Gross O, Netzer KO, Lambrecht R, Seibold S, Weber M. Meta-analysis of genotype-phenotype correlation in X-linked Alport syndrome: impact on clinical counselling. Nephrol Dial Transplant 2002;17:1218-1227.

[4] Jais JP, Knebelmann B, Giatras I, et al. X-linked Alport syndrome: natural history in 195 families and genotype- phenotype correlations in males. J Am Soc Nephrol 2000;11: 649-657.

[5] Lee JM, Nozu K, Choi DE, Kang HG, Ha IS, Cheong HI. Features of autosomal recessive Alport syndrome: a systematic review. J Clin Med 2019;8:178.

[6] Oka M, Nozu K, Kaito H, et al. Natural history of genetically proven autosomal recessive Alport syndrome. Pediatr Nephrol 2014;29:1535-1544.

[7] Jais JP, Knebelmann B, Giatras I, et al. X-linked Alport syndrome: natural history and genotype-phenotype correlations in girls and women belonging to 195 families: a "European Community Alport Syndrome Concerted Action" study. J Am Soc Nephrol 2003;14:2603-2610.

[8] Kamiyoshi N, Nozu K, Fu XJ, et al. Genetic, clinical, and pathologic backgrounds of patients with autosomal dominant Alport syndrome. Clin J Am Soc Nephrol 2016;11: 1441-1449.

[9] Yamamura T, Nozu K, Fu XJ, et al. Natural history and genotype-phenotype correlation in female X-linked Alport syndrome. Kidney Int Rep 2017;2:850-855.

[10] Yamamura T, Horinouchi T, Adachi T, et al. Development of an exon skipping therapy for X-linked Alport syndrome with truncating variants in COL4A5. Nat Commun 2020;11: 2777.

[11] Hashimura Y, Nozu K, Kaito H, et al. Milder clinical aspects of X-linked Alport syndrome in men positive for the collagen IV $\alpha 5$ chain. Kidney Int 2014;85:1208-1213.

[12] Said SM, Fidler ME, Valeri AM, et al. Negative staining for COL4A5 correlates with worse prognosis and more severe ultrastructural alterations in males with Alport syndrome. Kidney Int Rep 2016;2:44-52.

[13] Yamamura T, Nozu K, Minamikawa S, et al. Comparison between conventional and comprehensive sequencing approaches for genetic diagnosis of Alport syndrome. Mol Genet Genomic Med 2019;7:e883.

[14] Shono A, Tsukaguchi H, Kitamura A, et al. Predisposition to relapsing nephrotic syndrome by a nephrin mutation that interferes with assembly of functioning microdomains. Hum Mol Genet 2009;18:2943-2956.

[15] Imafuku A, Nozu K, Sawa N, et al. Autosomal dominant form of type IV collagen nephropathy exists among patients with hereditary nephritis difficult to diagnose clinicopathologically. Nephrology (Carlton) 2018;23:940-947.

[16] Horinouchi T, Nozu K, Yamamura T, et al. Detection of splicing abnormalities and genotype-phenotype correlation in X-linked Alport syndrome. J Am Soc Nephrol 2018; 29:2244-2254.

[17] Nagano C, Nozu K, Morisada N, et al. Detection of copy number variations by pair analysis using next-generation sequencing data in inherited kidney diseases. Clin Exp Nephrol 2018;22:881-888.

[18] Nozu K, Krol RP, Nakanishi K, et al. Detection by multiplex ligation-dependent probe amplification of large deletion mutations in the COL4A5 gene in female patients with $\mathrm{Al}-$ port syndrome. Pediatr Nephrol 2009;24:1773-1774.

[19] Nozu K, Vorechovsky I, Kaito H, et al. X-linked Alport syndrome caused by splicing mutations in COL4A5. Clin J Am Soc Nephrol 2014;9:1958-1964.

[20] Kamura M, Yamamura T, Omachi K, et al. Trimerization and genotype-phenotype correlation of COL4A5 mutants in Alport syndrome. Kidney Int Rep 2020;5:718-726.

[21] Omachi K, Kamura M, Teramoto K, et al. A split-luciferasebased trimer formation assay as a high-throughput screening platform for therapeutics in Alport syndrome. Cell Chem Biol 2018;25:634-643.e4. 
[22] Zhang Y, Ding J, Wang S, et al. Reassessing the pathogenicity of c.2858G >T(p.(G953V)) in COL4A5 gene: report of 19 Chinese families. Eur J Hum Genet 2020;28:244-252.

[23] Kaneko K, Tanaka S, Hasui M, et al. A family with X-linked benign familial hematuria. Pediatr Nephrol 2010;25:545548.

[24] Morizane R, Lam AQ, Freedman BS, Kishi S, Valerius MT, Bonventre JV. Nephron organoids derived from human pluripotent stem cells model kidney development and injury. Nat Biotechnol 2015;33:1193-1200.

[25] Takasato M, Er PX, Chiu HS, et al. Kidney organoids from human iPS cells contain multiple lineages and model human nephrogenesis. Nature 2015;526:564-568.

[26] Taguchi A, Kaku Y, Ohmori T, et al. Redefining the in vivo origin of metanephric nephron progenitors enables generation of complex kidney structures from pluripotent stem cells. Cell Stem Cell 2014;14:53-67.

[27] Takasato M, Er PX, Chiu HS, Little MH. Generation of kidney organoids from human pluripotent stem cells. Nat Protoc 2016;11:1681-1692.

[28] van den Berg CW, Ritsma L, Avramut MC, et al. Renal subcapsular transplantation of PSC-derived kidney organoids induces neo-vasculogenesis and significant glomerular and tubular maturation in vivo. Stem Cell Reports 2018;10:751765.

[29] Gao E, Yang X, Si N, Liu K, Wang JQ, Liu Z. A novel COL4A5 splicing mutation causes skipping of exon 14 in a Chinese family with Alport syndrome. Kidney Dis (Basel) 2020;6:4349.

[30] Horinouchi T, Nozu K, Yamamura T, et al. Determination of the pathogenicity of known COL4A5 intronic variants by in vitro splicing assay. Sci Rep 2019;9:12696.

[31] Malone AF, Funk SD, Alhamad T, Miner JH. Functional assessment of a novel COL4A5 splice region variant and immunostaining of plucked hair follicles as an alternative method of diagnosis in X-linked Alport syndrome. Pediatr Nephrol 2017;32:997-1003.

[32] Fu XJ, Nozu K, Eguchi A, et al. X-linked Alport syndrome associated with a synonymous p.Gly292Gly mutation alters the splicing donor site of the type IV collagen alpha chain 5 gene. Clin Exp Nephrol 2016;20:699-702.

[33] Horinouchi T, Yamamura T, Minamikawa S, et al. Pathogenic evaluation of synonymous COL4A5 variants in Xlinked Alport syndrome using a minigene assay. Mol Genet Genomic Med 2020;8:e1342.

[34] Iijima K, Nozu K, Kamei K, et al. Severe Alport syndrome in a young woman caused by a $\mathrm{t}(\mathrm{X} ; 1)(\mathrm{q} 22.3 ; \mathrm{p} 36.32)$ balanced translocation. Pediatr Nephrol 2010;25:2165-2170.

[35] Migeon BR. X inactivation, female mosaicism, and sex differences in renal diseases. J Am Soc Nephrol 2008;19:20522059.

[36] Storey H, Savige J, Sivakumar V, Abbs S, Flinter FA. COL4A3/COL4A4 mutations and features in individuals with autosomal recessive Alport syndrome. J Am Soc Nephrol 2013;24:1945-1954.

[37] Groopman EE, Marasa M, Cameron-Christie S, et al. Diagnostic utility of exome sequencing for kidney disease. $\mathrm{N}$ Engl J Med 2019;380:142-151.

[38] Imafuku A, Nozu K, Sawa N, Nakanishi K, Ubara Y. How to resolve confusion in the clinical setting for the diagnosis of heterozygous COL4A3 or COL4A4 gene variants? Discussion and suggestions from nephrologists. Clin Exp Nephrol 2020;24:651-656.

[39] Kashtan CE, Ding J, Garosi G, et al. Alport syndrome: a unified classification of genetic disorders of collagen IV $\alpha 345$ : a position paper of the Alport Syndrome Classification Working Group. Kidney Int 2018;93:1045-1051.

[40] Webb NJ, Lam C, Shahinfar S, et al. Efficacy and safety of losartan in children with Alport syndrome--results from a subgroup analysis of a prospective, randomized, placeboor amlodipine-controlled trial. Nephrol Dial Transplant 2011;26:2521-2526.

[41] Webb NJ, Shahinfar S, Wells TG, et al. Losartan and enalapril are comparable in reducing proteinuria in children with Alport syndrome. Pediatr Nephrol 2013;28:737-743.

[42] Gross O, Licht C, Anders HJ, et al. Early angiotensin-converting enzyme inhibition in Alport syndrome delays renal failure and improves life expectancy. Kidney Int 2012;81: 494-501.

[43] Gross O, Tönshoff B, Weber LT, et al.; German Pediatric Nephrology (GPN) Study Group and EARLY PRO-TECT Alport Investigators. A multicenter, randomized, placebocontrolled, double-blind phase 3 trial with open-arm comparison indicates safety and efficacy of nephroprotective therapy with ramipril in children with Alport's syndrome. Kidney Int 2020;97:1275-1286.

[44] Nangaku M, Kanda H, Takama H, Ichikawa T, Hase H, Akizawa T. Randomized clinical trial on the effect of bardoxolone methyl on GFR in diabetic kidney disease patients (TSUBAKI study). Kidney Int Rep 2020;5:879-890.

[45] Gomez IG, MacKenna DA, Johnson BG, et al. Anti-microRNA-21 oligonucleotides prevent Alport nephropathy pro- 
gression by stimulating metabolic pathways. J Clin Invest 2015;125:141-156.

[46] Hashikami K, Asahina M, Nozu K, Iijima K, Nagata M,
Takeyama M. Establishment of X-linked Alport syndrome model mice with a Col4a5 R471X mutation. Biochem Biophys Rep 2018;17:81-86. 